\title{
SUR UN THEORÈME DE G. SZEKERES, CONCERNANT LES FONGTIONS MONOTONES ET CONVEXES
}

\section{S. MARCUS}

1. Dans une note récente (1), Szekeres a donné le théorème suivant:

Si f est une fonction réelle, définie, strictement croissante et ayant la deuxième dérivée continue sur $(a, b)(-\infty \leqslant a<b \leqslant \infty)$, alors il existe une fonction réelle $\phi$, définie et strictement croissante sur $(a, b)$, et une fonction réelle $\psi$, définie et strictement croissante sur $(\phi(a), \phi(b))$, telles que $\phi^{\prime \prime}(x) \geqslant 0$ pour $a<x<b$, $\psi^{\prime \prime}(u) \leqslant 0$ pour $\phi(a)<u<\phi(b)$ et $f(x)=\psi(\phi(x))$ pour $a<x<b$.

Dans la démonstration de ce théorème, Szekeres utilise une expression où la dérivée $f^{\prime}(x)$ apparaît au dénominateur, ce qui exige qu'on ait $f^{\prime}(x) \neq 0$ pour $a<x<b$, condition qui ne figure pas dans l'énoncé du théorème. Il est naturel alors de poser le problème si le théorème ci-dessus reste en vigueur si cette condition n'est pas remplie. Il y a lieu aussi de se demander si les autres conditions, par exemple l'existence et la continuité de $f^{\prime \prime}(x)$ sont nécessaires pour qu'une fonction strictement croissante puisse être représentée comme une superposition de deux fonctions croissantes, l'une convexe, l'autre concave. Dans ce qui suit nous allons donner une réponse à ces questions. Nous allons voir non seulement que le théorème cesse d'être vrai si $f^{\prime}(x)$ s'annule (même en un seul point de $(a, b)$ ), mais qu'on a aussi d'autres conditions nécessaires, de nature assez restrictive, pour que $f$ admette une représentation du type que nous venons de parler. D'autre part, nous allons établir des conditions suffisantes assez voisines de celles nécessaires, de sorte qu'une amélioration de ces conditions ne paraît pas possible sans compliquer les hypothèses-hors mis le cas où les conditions nécessaires établies par le théorème 1 sont aussi suffisantes, question que nous ne savons pas résoudre.

2. Une fonction réelle $g$ est convexe (concave) sur $(a, b)$ si pour $a<x<y<b$ on a

$$
g\left(\frac{x+y}{2}\right) \leqslant \frac{g(x)+g(y)}{2}\left(g\left(\frac{x+y}{2}\right) \geqslant \frac{g(x)+g(y)}{2}\right) .
$$

Considérons une fonction réelle $f$, définie sur $(a, b)(-\infty \leqslant a<b \leqslant \infty)$. Supposons qu'il existe une fonction réelle $\phi$, définie, convexe et strictement croissante sur $(a, b)$ et une fonction réelle $\psi$, définie, concave et strictement croissante sur $(\phi(a), \phi(b))$, telles que

$$
f(x)=\psi(\phi(x)) \text { pour } a<x<b
$$

Nous dirons en ce cas que $f$ est une fonction $d u$ type $K$.

\footnotetext{
Reçu le 25 novembre, 1958.
} 
Si pour une fonction réelle $f$ il existe, en un point $x$, la dérivée à droite (gauche) finie, nous désignons cette dérivée par $f^{+}(x)\left(f^{-}(x)\right)$.

THÉORÈme 1. Si une fonction réelle $f$, définie sur $(a, b)$, est du type $K$ sur $(a, b)$, alors les fonctions $f^{+}(x)$ et $f^{-}(x)$ sont définies sur $(a, b)$. Chacune de ces deux fonctions est dérivable presque partout sur $(a, b)$ et la dérivée est sommable sur tout intervalle compact contenu dans $(a, b)$. Pour $a<x<b$ on a $f^{-}(x)>0<f^{+}(x)$.

Démonstration. Les fonctions $\phi$ et $\psi$, étant monotones, admettent des points de continuité donc, en vertu de la convexité de $\phi$ et de la concavité de $\psi, \phi$ et $\psi$ sont continues en chaque point de $(a, b)$ resp. $(\phi(a), \phi(b))$. D'après un théorème classique, $\phi^{-}(x)$ et $\phi^{+}(x)$ existent en chaque point de $(a, b)$, $\psi^{-}(u)$ et $\psi^{+}(u)$ existent en chaque point de $(\phi(a), \phi(b))$. On a donc, en vertu de la monotonie stricte de $\phi$,

$$
f^{-}(x)=\psi^{-}(\phi(x)) \cdot \phi^{-}(x), f^{+}(x)=\psi^{+}(\phi(x)) \cdot \phi^{+}(x) \text { si } a<x<b .
$$

En vertu de la convexité de $\phi, \phi^{-}$, et $\phi^{+}$sont des fonctions croissantes sur $(a, b)$. En vertu de la concavité de $\psi, \psi^{-}$et $\psi^{+}$sont des fonctions décroissantes sur $(\phi(a), \phi(b))$, donc $\psi^{-}(\phi(x))$ et $\psi^{+}(\phi(x))$ sont décroissantes sur $(a, b)$. Par suite, chacune des fonctions $f^{-}$et $f^{+}$est un produit de deux fonctions monotones, donc de deux fonctions dérivables presque partout. Il s'ensuit que $f^{-}$ et $f^{+}$sont dérivables presque partout sur $(a, b)$.

Soit maintenant $\langle\alpha, \beta\rangle$ un intervalle compact contenu dans $(a, b) . \psi^{-}(x)$, $\psi^{-}(\phi(x)), \phi^{+}(x)$ et $\psi^{+}(\phi(x))$ sont monotones et bornées sur $\langle\alpha, \beta\rangle$, donc $f^{-}$ et $f^{+}$sont à variation bornée sur $\langle\alpha, \beta\rangle$. Mais d'après un théorème bien connu, telles fonctions admettent des dérivées sommables sur $\langle\alpha, \beta\rangle$.

$f$, comme superposition de fonctions strictement croissantes, est strictement croissante sur $(a, b)$ donc, en vertu des théorèmes connus (par exemple celui de Scheefer $\left(2\right.$, p. 138)) $f^{+}$ne peut s'annuler identiquement en aucun intervalle. Il s'ensuit que $\phi^{+}(x)$ et $\psi^{+}(\phi(x))$ ne peuvent s'annuler identiquement en aucun intervalle contenu dans $(a, b)$ donc $\phi^{+}$, qui d'après le raisonnement ci-dessus est croissante sur $(a, b)$, est même strictement croissante sur $(a, b)$. On constate aussi que $\psi^{+}(\phi(x))$ est strictement décroissante. D'autre part, en vertu de la monotonie croissante des fonctions $\phi$ et $\psi$, on a $\phi^{+}(x)$ $\geqslant 0 \leqslant \psi^{+}(\phi(x))$ pour $a<x<b$ et, en vertu de la monotonie stricte de $\phi^{+}(x)$ et $\psi^{+}(\phi(x))$, que nous venons d'établir, on conclut: $\phi^{+}(x)>0<\psi^{+}(\phi(x))$ pour $a<x<b$.

D'une manière analogue on prouve que $\phi^{-}(x)>0<\psi^{-}(\phi(x))$ pour $a<x<b$ et le théorème 1 est complètement démontré.

Un exemple d'une fonction $f$ qui satisfait toutes les hypothèses du théorème de Szekeres mais ne remplit pas la condition $f^{-}(x) \neq 0 \neq f^{+}(x)$ est donné par $f(x)=x^{3}$, considérée sur n'importe quel intervalle ouvert contenant l'origine. En effet, on a $f^{\prime}(0)=0$. Donc, en vertu du théorème $1, f(x)=x^{3}$ n'est du 
type $K$ sur aucun intervalle $(a, b)$ tel que $a<0<b$, c'est-à-dire cette fonction ne vérifie pas la thèse du théorème de Szekeres.

3. Maintenant se pose le problème: les conditions nécessaires données par le théorème 1 sont-elles aussi suffisantes pour que la fonction $f$ soit du type $K$ ? Nous ne savons pas. Mais nous allons montrer qu'il suffit d'ajouter, aux conditions nécessaires établies par le théorème 1 , la condition que $f^{+}$soit dérivable non seulement presque partout, mais partout sur $(a, b)$, pour que $f$ soit du type $K$.

LEмме. Si $g$ est une fonction réelle croissante et absolument continue sur $(a, b)$ et si $\phi$ est une fonction réelle absolument continue sur $(g(a), g(b))$, alors la fonction $\lambda$ donnée par $\lambda(x)=\phi(g(x))$ est absolument continue sur $(a, b)$.

Nous laissons au lecteur la démonstration de ce lemme.

Rappelons qu'une fonction $f$ est absolument continue au sens généralisé sur $(a, b)$, si elle est continue sur $(a, b)$ et s'il existe une décomposition $(a, b)$ $=\infty^{\prime} E_{n}$, où $E_{n}$ sont des ensembles tels que $f$ soit absolument continue sur $E_{n}$, quel que soit $n$ (3, p. 223).

THÉoRème 2 . Soit f une fonction réelle dénbie sur $(a, b)(-\infty \leqslant a<b \leqslant \infty)$. Supposons que $f$ est dérivable sur $(a, b)$ et que $f^{\prime}(x)>0$ pour $a<x<b$. Supposons encore que $f^{\prime \prime}(x)$ existe, finie, en chaque point de $(a, b)$ et que $f^{\prime \prime}$ est sommable sur chaque intervalle compact contenu dans $(a, b)$. Alors, $f$ est, sur $(a, b)$, une fonction du type $K$.

Remarque. Pour comprendre la situation du theorème 2 par rapport au théorème 1 il faut rappeler le théorème de Dini, d'après lequel, si $f^{+}$est continue en $x$, alors $f^{\prime}(x)$ existe et est finie (voir, par exemple, (3, p. 204)).

Démonstration du théorème 2 . On va utiliser l'idée de la démonstration du théorème 1 de (1).

La fonction

$$
f_{+}^{\prime \prime}(x)=\left\{\begin{array}{l}
f^{\prime \prime}(x) \text { si } f^{\prime \prime}(x)>0 \\
0 \text { si } f^{\prime \prime}(x) \leqslant 0
\end{array}\right.
$$

est sommable sur chaque intervalle compact contenu dans $(a, b)$. Par hypothèse, $f^{\prime}(x)$ est continue et strictement positive sur $(a, b)$. Il existe donc pour chaque intervalle compact $J \subset(a, b)$ un nombre $\rho(J)>0$ tel que

$$
\frac{1}{\rho(J)}<f^{\prime}(x)<\rho(J)
$$

pour $x \in J$. Il s'ensuit donc que l'intégrale

$$
p(y)=\int_{d}^{y} \frac{f_{+}^{\prime \prime}(x)}{f^{\prime}(x)} d x
$$

où $d$ est un point fixe de $(a, b)$, existe, au sens de Lebesgue, pour chaque $y$ tel que $a<y<b$. 
La fonction donnée par $p(y)$ est continue, strictement croissante et strictement positive sur $(a, b)$. Il s'ensuit donc que la fonction

$$
\phi(x)=\int_{d}^{x} e^{p(y)} d y
$$

où l'intégrale est prise au sens de Cauchy, est strictement croissante et convexe sur $(a, b)$.

Désignons par $\psi$ l'inverse de la fonction $\phi . \psi$ est une fonction définie sur $(\phi(a), \phi(b))$, strictement croissante et concave sur $(\phi(a), \phi(b))$ et ayant les valeurs dans $(a, b)$. Le théorème 2 sera démontré dès qu'on aura prouvé que la fonction donnée par

$$
h(u)=f(\psi(u))
$$

est concave sur $(\phi(a), \phi(b))$, car on a en tout cas, en vertu de ce que $\psi$ et $\phi$ sont des fonctions inverses,

$$
h(\phi(x))=f(\psi(\phi(x)))=f(x) .
$$

Mais $\phi$ est une fonction dérivable, à dérivée strictement positive sur $(a, b)$, donc $\psi$ est dérivable sur $(\phi(a), \phi(b))$. Il s'ensuit que $h$ est une fonction dérivable sur $(\phi(a), \phi(b))$. Pour montrer que $h$ est concave, il suffit de montrer que $h^{\prime}(u)$ est décroissante sur $(\phi(a), \phi(b))$. Le théorème sera donc démontré si nous pourrons montrer que

$1^{\circ} h^{\prime}(u)$ est absolument continue sur tout intervalle compact contenu dans $(\phi(a), \phi(b))$;

$2^{\circ} h^{\prime \prime}(u) \leqslant 0$ presque partout sur $(\phi(a), \phi(b))$.

En effet, dans ce cas $h^{\prime}(u)$ sera absolument continue au sens généralisé $\operatorname{sur}(\phi(a), \phi(b))$. Mais on sait d'après un théorème classique (voir, par exemple, (3, p. 223)) qu'une fonction absolument continue au sens généralisé est décroissante dès que sa dérivée approximative est presque partout $\leqslant 0$.

Démontrons donc, $1^{\circ}$ et $2^{\circ}$. Remarquons d'abord que la dérivée de $\phi$ est une fonction absolument continue sur tout intervalle compact $J$ contenu dans $(a, b)$. En effet, on a pour chaque $x \in(a, b), \phi^{\prime}(x)=e^{p(x)}$, où $p(x)$ est croissante et, étant une intégrale de Lebesgue, est absolument continue sur $J$.

Remarquons ensuite que la borne inférieure de $\phi^{\prime}(x)$ sur $J$ est strictement positive. D'autre part, $\psi$ étant continue et concave, est absolument continue sur $\phi(J)$. En tenant compte que $\psi$ est croissante, on déduit, en vertu du lemme, que $\phi^{\prime}(\psi(u))$ est absolument continue et strictement positive sur $\phi(J)$. Il s'ensuit donc que

$$
\psi^{\prime}(u)=\frac{1}{\phi^{\prime}(\psi(u))}
$$

est aussi absolument continue sur $\phi(J)$, qui peut être considéré comme un intervalle compact quelconque contenu dans $(\phi(a), \phi(b)) . f^{\prime}(x)$, ayant une dérivée finie sur $J$, est absolument continue au sens généralisé sur $J$. La fonction

$$
F(x)=\int_{\alpha}^{x} f^{\prime \prime}(u) d u
$$


où $\alpha$ est l'extrémité gauche de $J$, est absolument continue sur $J$. Mais les dérivées des fonctions $f^{\prime}(x)$ et $F(x)$ coïncident presque partout sur $J$ donc, en vertu d'un théorème connu $(3$, p. 225) ces fonctions diffèrent par une constante. Il s'ensuit que $f^{\prime}(x)$ est absolument continue sur $J$.

$\psi$, étant continue et concave, est absolument continue sur tout intervalle compact contenu dans $(\phi(a), \phi(b))$. En vertu de ce que $\psi$ est croissante, on peut faire usage du lemme et conclure que $f^{\prime}(\psi(u))$ est absolument continue sur $\phi(J)$. De ce que $h^{\prime}(u)=f^{\prime}(\psi(u)) \cdot \psi^{\prime}(u)$ on déduit alors que $h^{\prime}$ est absolument continue sur $\phi(J)$.

Démontrons maintenant que $h^{\prime \prime}(u) \leqslant 0$ presque partout sur $(\phi(a), \phi(b))$. L'existence, presque partout, de $h^{\prime \prime}(u)$, est assurée par le fait que $h^{\prime}(u)$, étant absolument continue, est à variation bornée sur tout intervalle compact contenu dans $(\phi(a), \phi(b))$. On doit montrer que

$$
h^{\prime \prime}(u)=f^{\prime \prime}(\psi(u)) \cdot \psi^{12}(u)+f^{\prime}(\psi(u)) \cdot \psi^{\prime \prime}(u) \leqslant 0
$$

presque partout sur $(\phi(a), \phi(b))$. Mais on a

$$
\psi^{\prime \prime}(u)=-\frac{\phi^{\prime \prime}(\psi(u))}{\phi^{\prime 3}(\psi(u))} .
$$

En posant $x=\psi(u)$ et en remarquant que chacune des fonctions $\phi$ et $\psi$, en vertu de leur continuité absolue, transforme chaque ensemble de mesure nulle dans un ensemble de mesure nulle (3, p. 225) l'inégalité ci-dessus devient

$$
\frac{f^{\prime \prime}(x)}{\phi^{\prime 2}(x)}-f^{\prime}(x) \frac{\phi^{\prime \prime}(x)}{\phi^{\prime 3}(x)} \leqslant 0
$$

presque partout sur $(a, b)$, donc

$$
\frac{f^{\prime \prime}(x)}{f^{\prime}(x)} \leqslant \frac{\phi^{\prime \prime}(x)}{\phi^{\prime}(x)}
$$

presque partout sur $(a, b)$. Mais cette inégalité est évidente, car on a, presque partout sur $(a, b)$,

$$
\frac{\phi^{\prime \prime}(x)}{\phi^{\prime}(x)}=\frac{f_{+}^{\prime \prime}(x)}{f^{\prime}(x)}
$$

et, d'autre part, pour chaque $x \in(a, b)$,

$$
\frac{f_{+}^{\prime \prime}(x)}{f^{\prime}(x)} \geqslant \frac{f^{\prime \prime}(x)}{f^{\prime}(x)}
$$

Le théorème 2 est ainsi complètement démontré.

Remarque. L'hypothèse que $f^{\prime \prime}(x)$ existe, finie, non seulement presque partout, mais partout sur $(a, b)$, a été posée seulement pour assurer la continuité absolue de $f^{\prime}(x)$ sur chaque intervalle compact contenu dans $(a, b)$. 


\section{LITTÉRATURE}

1. G. Szekeres, On a property of monotone and convex functions, Proc. Amer. Math. Soc., 7 (1956), 351-3.

2. S. Saks, Théorie de l'intégrale (Warszawa, 1933).

3. - Theory of the integral (Warszawa-Lwow, 1937).

\section{Institut de Matematicā}

București 